\title{
Prognostic values of the core components of the mammalian circadian clock in prostate cancer
}

\author{
Wenchang Yue ${ }^{\text {Equal first author, } 1}{ }^{1}$, Xiao Du ${ }^{\text {Equal first author, } 2}{ }^{2}$ Xuhong Wang ${ }^{3}$, Niu Gui ${ }^{4}$, Weijie Zhang ${ }^{1}$, Jiale Sun ${ }^{1}$, Jiawei You ${ }^{1}$, \\ Dong He ${ }^{1}$, Xinyu Geng ${ }^{1}$, Yuhua Huang ${ }^{1}$, Jianquan Hou ${ }^{\text {Corresp. } 1}$ \\ ${ }^{1}$ Department of Urology, The First Affiliated Hospital of Soochow University, Suzhou, China \\ 2 Department of Radiation Oncology, The First Affiliated Hospital of Soochow University, Suzhou, China \\ 3 Department of Urology, Tongcheng people's Hospital, Tongcheng, China \\ 4 General Surgery Ward 2, Fengtaixian Hospital of Chinese Medicine, Huainan, China \\ Corresponding Author: Jianquan Hou \\ Email address: change920@163.com
}

Background: Prostate cancer (PC) is one of the most common malignancies in males. Extensive and complex connections between circadian rhythm and cancer were found. Nonetheless, in PC, the potential role of the core components of the mammalian circadian clock (CCMCCS) in prognosis prediction has not been fully clarified. Methods: We firstly collected 605 patients with PC from the Cancer Genome Atlas (TCGA) and the Gene Expression Omnibus (GEO) Databases. Survival analysis was carried out for each CCMCC. Then, we investigated the prognostic ability of CCMCCs by Cox regression analysis. Independent prognostic signatures were extracted for the establishment of the circadian clock-based risk score model. We explored the predictive performance of the risk score model in the TCGA training cohort and the independent GEO dataset. Finally, the relationships between risk score and clinicopathological parameters, biological processes, and signaling pathways were evaluated. Results: The expression levels of CCMCCs were widely correlated with age, tumor status, lymph node status, disease-free survival (DFS), progression-free survival (PFS), and overall survival (OS). Nine circadian clock genes, including CSNK1D, BTRC, CLOCK, CSNK1E, FBXL3, PRKAA2, DBP, NR1D2, and RORB, were identified as vital prognostic factors in $\mathrm{PC}$ and were used to construct the circadian clockbased risk score model. For DFS, the area under the 3-year or 5-year receiver operating characteristic curves ranged from 0.728 to 0.821 , suggesting better predictive performance. When compared with T3-4N1 stage, PC patients at T2N0 stage might be benefited more from the circadian clock-based risk score model. Furthermore, a high circadian clock-based risk score indicated shorter DFS $(p<0.0001)$, early progression $(p<0.0001)$, and higher 5 -year death rate $(p=0.007)$ in PC. The risk score was related to tumor status $(p<0.001)$, lymph node status $(p<0.001)$, and ribosome-related biogenesis and pathways. Conclusions: The vital roles of circadian clock genes in clinical outcomes Peerr reviewing PDF | (2021:06:63103:1:1:NEW 4 Oct 2021) 
were fully depicted. The circadian clock-based risk score model could reflect and predict the prognosis of patients with PC. 
1 Prognostic values of the core components of the mammalian circadian clock in

3 Wenchang Yue ${ }^{\# 1}$, Xiao Du ${ }^{\# 2}$, Xuhong Wang ${ }^{3}$, Niu Gui ${ }^{4}$, Weijie Zhang ${ }^{1}$, Jiale Sun ${ }^{1}$,

4 Jiawei You ${ }^{1}$, Dong $\mathrm{He}^{1}$, Xinyu Geng ${ }^{1}$, Yuhua Huang ${ }^{1}$, Jianquan $\mathrm{Hou}^{1 *}$

5 1. Department of Urology, The First Affiliated Hospital of Soochow University,

$6 \quad$ Suzhou 215006, People's Republic of China.

7 2. Department of Radiation Oncology, The First Affiliated Hospital of Soochow

$8 \quad$ University, Suzhou 215006, People's Republic of China.

9 3. Department of Urology, Tongcheng people's Hospital, Tongcheng 231400,

10 People's Republic of China.

4. General Surgery Ward 2, Fengtaixian Hospital of Chinese Medicine, Huainan 232100, People's Republic of China.

\#Wenchang Yue and Xiao Du contributed equally to this work and shall share first authorship.

$16 *$ correspondence author

Corresponding authors: Jianquan $\mathrm{Hou}$

Department of Urology, The First Affiliated Hospital of Soochow University,

Suzhou 215006, People's Republic of China.

Email address: change920@163.com 


\section{Abstract}

Background: Prostate cancer (PC) is one of the most common malignancies in males. Extensive and complex connections between circadian rhythm and cancer were found. Nonetheless, in PC, the potential role of the core components of the mammalian circadian clock (CCMCCs) in prognosis prediction has not been fully clarified.

Methods: We firstly collected 605 patients with PC from the Cancer Genome Atlas (TCGA) and the Gene Expression Omnibus (GEO) Databases. Survival analysis was carried out for each CCMCC. Then, we investigated the prognostic ability of CCMCCs by Cox regression analysis. Independent prognostic signatures were extracted for the establishment of the circadian clock-based risk score model. We explored the predictive performance of the risk score model in the TCGA training cohort and the independent GEO dataset. Finally, the relationships between risk score and clinicopathological parameters, biological processes, and signaling pathways were evaluated.

Results: The expression levels of CCMCCs were widely correlated with age, tumor status, lymph node status, disease-free survival (DFS), progression-free survival (PFS), and overall survival (OS). Nine circadian clock genes, including CSNK1D, BTRC, CLOCK, CSNK1E, FBXL3, PRKAA2, DBP, NR1D2, and RORB, were identified as vital prognostic factors in PC and were used to construct the circadian 
43 clock-based risk score model. For DFS, the area under the 3-year or 5-year receiver

44 operating characteristic curves ranged from 0.728 to 0.821 , suggesting better

45 predictive performance. When compared with T3-4N1 stage, PC patients at T2N0

46 stage might be benefited more from the circadian clock-based risk score model.

47 Furthermore, a high circadian clock-based risk score indicated shorter DFS

$48(\mathrm{p}<0.0001)$, early progression $(\mathrm{p}<0.0001)$, and higher 5-year death rate $(\mathrm{p}=0.007)$ in

49 PC. The risk score was related to tumor status $(\mathrm{p}<0.001)$, lymph node status

$50 \quad(\mathrm{p}<0.001)$, and ribosome-related biogenesis and pathways.

51 Conclusions: The vital roles of circadian clock genes in clinical outcomes were fully

52 depicted. The circadian clock-based risk score model could reflect and predict the 53 prognosis of patients with PC.

Key words: prostate cancer; core components of the mammalian circadian clock

56 (CCMCCs); prognosis; survival; risk score model

\section{Introduction}

59 Prostate cancer (PC) is the most common malignant tumor in the male urinary system (1). An increased incidence rate of PC was found around the world (1-3).

61 The incidence and mortality rates of PC reached up to 10 per 100,000 and 4 per 62100,000 , respectively (1-3). Individual differences of PC are obvious. For PC, the 63 therapeutic scheme of each patient mainly depends on tumor grade, doctors' clinical 
64 judgment, and conventional risk assessment. Conventional risk assessment included

65 several clinical factors: prostate specific antigen (PSA) level, TNM staging, and

66 Gleason score. Prostatectomy and radiotherapy are the recommended treatments for

67 localized PC. Even the prognosis of most clinical patients with early-stage PC was

68 satisfactory, postoperative recurrence was unavoidable (4). For advanced PC

69 patients, despite initial sensitivity to androgen deprivation therapy, the majority of

70 patients with PC finally developed resistance to castration therapy after 18 to 24

71 months of clinical treatment (4-6). Thus, it is of significance to identify patients with

72 high relapse risk.

74 The discoveries in the circadian rhythm were considered as dramatic breakthroughs

75 in the field of medicine. In 2017, three scientists won the Nobel Prize for their work

76 on the circadian rhythm $(7,8)$. Several studies highlighted the essential role of

77 circadian disruption in multiple biomolecular processes of cancer, including PC and

78 other solid tumors (9-16). The core components of the mammalian circadian clock

79 (CCMCCs) were defined as a group of genes that could regulate human circadian

80 rhythm through regulating RNA expression levels and biological pathways $(17,18)$.

81 CCMCCs are composed of a total of 22 genes. These 22 CCMCCs included 7 core

82 clock genes (CLOCK, ARNTL, PER1, PER2, PER3, CRY1) and 15 other circadian

83 clock-related genes (CRY2, BTRC, CSNK1D, CSNK1E, CUL1, DBP, FBXL21,

FBXL3, NFIL3, NR1D1, NR1D2, PRKAA1, PRKAA2, RORA, RORB, SKP1). 
85 CCMCCs predominately promoted many biochemical activities to work in rule and

86

87 order, thereby maintaining homeostasis. These key circadian clock genes also affected tumorigenesis, tumor growth, metastasis, and clinical outcomes of cancer patients. In tumor-bearing mice, the expression levels of five CCMCCs, including NR1D1, PER1, PER2, ARNTL, and DBP, were downregulated in hepatic metastasis from colorectal cancer when compared with healthy tissue (16). In lung cancer, both PER2 and ARNTL were tumor suppressor genes (13). In PC, overexpression of the clock gene PER1 promoted tumor cell apoptosis (19). The complex physically interaction between PER1 and the androgen receptor was also found (19). However, there are very limited researches that investigated the vital functions of key circadian clock genes in the pathogenesis and prognosis of PC. In this study, we systematically explored the association between circadian clock and prognosis in PC. Then, we proposed the circadian clock-based risk score and constructed a circadian clockrelated prognostic model. The performance of the risk score model was verified in the Cancer Genome Atlas (TCGA) dataset and the independent Gene Expression Omnibus (GEO) dataset. We found correlations between the circadian clock gene signature and several biological functions, signaling pathways, and clinicopathologic features.

Materials and methods

Dataset acquisition from the TCGA and GEO Database 
106 We identified suitable public datasets of PC patients in the TCGA (https ://tcga107 data.nci.nih.gov/tcga/) and GEO (https://www. ncbi. nlm. nih. gov/ geo/) Databases. 108 We eliminated datasets without intact gene expression data and prognostic 109 information. Both RNA sequencing (RNA-seq) data and complete clinical 110 annotation for each PC patient were downloaded online. In total, GSE70770 with 111112 PC patients (20) and TCGA-PC dataset with 493 PC patients (21) were 112 eventually gathered in this study for further analysis. Characteristics of the TCGA 113 cohort and the GEO cohort were summarized in Table S1.

\section{Construction of prognostic signature based on clock genes}

A total of 22 CCMCCs were obtained from previously published reviews $(17,18)$.

We applied the COX regression analysis to assess the effects of CCMCCs on clinical prognosis. We selected potential prognosis-related clock genes to construct the circadian clock-based risk score and the prognosis prediction model. The definition of the circadian clock-based risk score was as follows:

The circadian clock-based risk score $=\Sigma \lambda \mathrm{i}$

where $\mathrm{i}$ represents the expression of prognosis-related clock genes, $\lambda$ is the 123 coefficient that extracted from the COX regression analysis. The final formula of the 124 circadian clock-based risk score was as follows: the circadian clock-based risk score $125=(0.8 *$ expression value of CSNK1D $)+(-1.824 *$ expression value of BTRC $)+(-$ 
$127(1.239 *$ expression value of FBXL3) $+(-1.56 *$ expression value of PRKAA2 $)+$ $128\left(1.325^{*}\right.$ expression value of $\left.\mathrm{DBP}\right)+\left(0.433^{*}\right.$ expression value of NR1D2 $)+$ $129(1.049 *$ expression value of RORB). The training subset and the internal validation 130 subset was from the TCGA cohort, while GSE70770 was used as an external 131 validation dataset. The receiver operating characteristic (ROC) analysis was applied 132 to validate the performance of the proposed model. By $\mathrm{R}$ package termed 133 "survivalROC", areas under the ROC curve (AUC) were calculated.

\section{Functional enrichment analysis}

136 Differentially expressed genes (DEGs) between the high and low circadian clock137 based risk score groups were recognized by the "limma" $\mathrm{R}$ package. DEGs with 138 absolute value change of expression more than 2 and $p$ value less than 0.05 were 139 selected for signal pathway analysis. The Gene Ontology (GO, 140 http://www.geneontology.org/) and the Kyoto Encyclopedia of Genes and Genomes 141 (KEGG, https://www.kegg.jp/kegg/) enrichment analyses were utilized to reveal 142 unique biological processes and signal pathways between the high and low circadian 143 clock-based risk score groups.

Statistical analysis

146 The survival analysis of each CCMCC and the circadian clock-based risk score was 147 conducted by the Kaplan-Meier (KM) method and the log-rank test. The optimal 
148 cutoff point was evaluated by the R package "survminer". The differences between 149 the two groups were compared with the t-test. All statistical analyses were two150 tailed. And p value less than 0.05 was considered as statistical significance. The 151 visualization of all statistical results was performed by the SPSS 22.0 software and 152 the R 4.0.1 software.

\section{Results}

\section{Expression profile of CCMCCs in PC}

156 157 158 159 160 161 162 163 164 165 166 167 168

In the TCGA dataset, the gene expression of 22 CCMCCs and clinical features of 493 PC patients from TCGA database were summarized in Figure 1A and Table S1. Most subjects were under the age of 65 (327/493, 66.3\%). In addition, a majority of patients in the TCGA cohort were at N0 stage (342/493, 69.4\%). Pathology T3-4 patients also accounted for a large proportion $(300 / 493,60.9 \%)$. The number of patients who received neoadjuvant therapy was also summarized. We further explored the association between CCMCCs' expression levels and clinicopathological characteristics. Importantly, the expression level of SKP1 was downregulated in PC patients over 65 years old (Figure 1B). For lymph node (N) stage, CRY1 $(p<0.006), \quad$ CSNK1D $\quad(p<0.0014), \quad$ EBXL21 $\quad(p<0.001), \quad$ PER1 $(p<0.001)$, PER2 $(p<0.012)$, PRKAA2 $(p<0.011)$, and SKP1 $(p<0.007)$ were differentially expressed between the N0 and the N1 groups (Figure 1C). Moreover, for tumor (T) stage, ten CCMCCs differentially expressed between the T2 group and 
169 the T3-4 group (all $\mathrm{p}<0.05$, Figure 1D). In addition, relative high expression levels 170 of BTRC, CRY2, FBXL21, PER1, PER2, and PRKAA2, were found in the T2N0 171 group when compared with the T3-4N1 group (both $\mathrm{p}<0.05$, Figure $\mathrm{S} 1$ ). In the GEO 172 cohort, we further conducted correlation analysis on Gleason grade, PSA level, and 173 expression levels of 22 CCMCCs (Figure S2). However, regardless of statistical 174 correlation between Gleason grade and NR1D2 $(\mathrm{p}=0.011, \mathrm{r}=0.241)$, CLOCK $175(\mathrm{p}=0.045, \mathrm{r}=-0.192)$, as well as SKP1 $(\mathrm{p}=0.320, \mathrm{r}=-0.205)$, the correlation was weak 176 (Figure S2). No statistic relationship was found between PSA level, and expression 177 levels of 22 CCMCCs (Figure S2).

178

\section{Relationship between CCMCCs and prognosis}

To evaluate the association between CCMCCs expression and clinical outcomes, we performed the survival analysis. In the TCGA cohort, the sample size of prostate cancer patients who owned disease-free survival (DFS), progression-free survival 183 (PFS), and overall survival (OS) data was 333, 493, and 493, respectively (Figures 184 2-6). We found that high expression levels of ARNTL ( $p=0.037)$, CLOCK $(p=0.006), \quad$ PER2 $\quad(p=0.0051), \quad$ PER3 $\quad(p=0.013), \quad$ BTRC $\quad(p=0.026)$, CUL1 $(\mathrm{p}=0.0054)$, FBXL21 $(\mathrm{p}=0.00035)$, FBXL3 $(\mathrm{p}=0.012)$, PRKAA1 $(\mathrm{p}=0.013)$

187 PRKAA2 $(\mathrm{p}=0.015)$, and RORB $(\mathrm{p}=0.0067)$ were related to longer DFS (Figures 2 188 and 3). On the contrary, overexpression of CRY1 ( $p=0.0015)$, CSNK1D 189 $(p=0.00032), \operatorname{CSNK} 1 \mathrm{E}(\mathrm{p}=0.0019), \mathrm{DBP}(\mathrm{p}=0.00075), \mathrm{NR} 1 \mathrm{D} 1(\mathrm{p}=0.04)$, and SKP1 
$(p=0.017)$ were associated with poor DFS (Figures 2 and 3). For PFS, KM survival 191 analysis found that PFS was statistically related to the expression levels of 18 192 CCMCCs (Figures 4 and 5). In addition, there were high connections between the 193 expression levels of CSNK1D ( $p=0.016)$, FBXL21 $(p=0.039)$, NFIL3 $(p=0.037)$, PER1 ( $\mathrm{p}=0.035), \operatorname{RORB}(\mathrm{p}=0.037)$ and OS in KM curves (Figure 6).

Considering the influence of TNM stage on prognosis, we applied survival subgroup 197 analysis to the T2N0 cohort and the T3-4N1 cohort (Tables S2-S7). Importantly, at both T2N0 stage and T3-4N1 stage, high BTRC, CLOCK, CRY1, FBXL3, PER3, and RORA expression indicated longer DFS (both $\mathrm{p}<0.05$, Tables $\mathrm{S} 2-\mathrm{S} 3$ ). It was 200 also worth to mention that high DBP linked to shorter PFS in T2N0 prostate cancer 201 $(p=0.013$, Table S54), while the contrary result was found in T3-4N1 stage patients 202 $(p=0.0016$, Table S5). For OS, high NR1D1 expression was only significantly 203 related to better prognosis in T2N0 stage patients $(\mathrm{p}=0.041$, Table S6), while the 204 negative result was found in T3-4N1 stage patients ( $p=0.13$, Table S7). 205 206

\section{Identification of potential prognostic CCMCCs}

207 In the univariate Cox regression analysis, BTRC $(\mathrm{p}=0.020136)$, CLOCK $208 \quad(p=0.037606), \quad$ CSNK1D $\quad(p=0.002343), \quad$ CSNK1E $\quad(p=0.006002), \quad$ FBXL3 $209(\mathrm{p}=0.01807)$, and PRKAA2 $(\mathrm{p}=0.028131)$ were significantly associated with DFS 210 (Table 1). Additionally, among 22 CCMCCs, six genes, including CLOCK 
$211 \quad(p=0.042614), \operatorname{CSNK} 1 \mathrm{E}(\mathrm{p}=0.029721), \mathrm{DBP}(\mathrm{p}=0.002931), \mathrm{NR} 1 \mathrm{D} 2(\mathrm{p}=0.041884)$,

212 PRKAA2 ( $\mathrm{p}=0.018837)$, and RORB ( $\mathrm{p}=0.00098)$, showed significant relationship

213 with PFS (Table 2). For OS, CSNK1D was found to be potential prognostic factor

$214(\mathrm{p}=0.000651$, Table 3). After combining above findings and removing repetitive

215 gene, 9 CCMCCs were left, including CSNK1D, BTRC, CLOCK, CSNK1E,

216 FBXL3, PRKAA2, DBP, NR1D2, and RORB. We proposed a hypothesis that 9

217 CCMCCs were key prognostic genes in PC and incorporating them could effectively 218 predict the prognosis of PC patients.

219

220

Construction and validation of circadian clock-based risk score

221

To verify our hypothesis, we separated the TCGA cohort into the test set and the

222

internal validation set in a ratio of 7:3. All enrolled patients in the cohort underwent

223

surgical treatment, thus we mainly investigated the application of the predictive

224

model in DFS prediction. As shown in figures 7A-B, the AUC value of the predictive

225

model in the training cohort and the validation cohort for 3-year DFS was 0.742 and

226

0.821 , respectively. The ROC curves and AUC values $(0.728$ for the test cohort;

227

0.753 for the validation cohort) further indicated the satisfactory predictive power

228

of the circadian clock-based signature in DFS prediction (Figures 7C-D). PC patients

229

with high circadian clock-based risk score also had lower DFS than the low risk

230

score group ( $\mathrm{p}<0.0001$, Figure 7E). In an independent cohort with $112 \mathrm{PC}$ patients

231

who prostatectomy, we divided the GSE70770 cohort into high and low risk score 
232 groups and conducted the KM survival analysis. Results showed that the high 233 circadian clock-based risk score was correlated with early relapse (vs low circadian 234 clock-based risk score: 13.000 months vs 21.000 months, $p=0.06$; Figure S3).

Moreover, we explored the impact of TNM stage. When compared with clinical 237 prognostic factors, such as $\mathrm{T}$ (AUC values range from 0.502 to 0.808 ) and $\mathrm{N}$ stage 238 (AUC values range from 0.500 to 0.515 ), the predictive performance of the proposed 239 model was superior in DFS (Figure S4). Similar results were also detected in PFS 240 and OS prediction (Figure S4). The improvement of utility when combined circadian 241 clock-based risk score model with $\mathrm{T}$ and $\mathrm{N}$ stage was limited. Then, for better 242 clinical application, we investigated the power of circadian clock-based risk score 243 model in T2N0 disease and T3-4N1 disease (Figure S5). AUC values of DFS curves 244 indicated that the risk model performed better in patients with T2N0 stage (vs T3245 4N1 stage, AUC value: $0.749-0.834$ vs $0.515-0.745$, Figure S5). For PFS, the 246 predictive values of risk score model in T2N0 disease and T3-4N1 disease was 247 almost (Figure S5). In T2N0 stage, high risk score was significantly related to shorter 248 DFS ( $\mathrm{p}=0.0024)$ and PFS $(\mathrm{p}=0.034)$, while no statistical significance was found in OS ( $p=0.22$, Figure S6). In T3-4N0 stage, high circadian clock-based risk score was 250 significantly related to shorter PFS ( $\mathrm{p}=0.016)$, while no statistical significance was 251 found in DFS $(\mathrm{p}=0.12)$ and $\mathrm{OS}(\mathrm{p}=0.24$, Figure $\mathrm{S} 6)$. 
253 We also tentatively applied the risk score model to PFS and OS prediction (Figures

254 S7-8). The 3-year and 5-year AUC values of PFS curves ranged from 0.607-0.735

255 (Figure S7), while the AUC values of OS curves were higher than 0.700 (Figure S8).

256 The high circadian clock-based risk score indicated poor PFS ( $\mathrm{p}<0.0001)$, and higher

$257 \quad 5$-year death rate $(\mathrm{p}=0.007)$.

258

259

Correlation between clinicopathological parameters and circadian clock-based

260

risk score

261

The relationship between clinicopathological parameters and circadian clock-based

262

risk scores was evaluated in the TCGA cohort. There was no significant difference

263

in the risk score according to age $(\mathrm{p}=0.19$; Figure S9A). However, high risk score

264

was significantly linked to high $T$ status $(p=0.00015)$ and $N$ status $(p=0.00051$;

265

Figures S9B-C). When in comparison with T2N0 stage, we found that higher

266

circadian clock-based risk score was found in the T3-4N1 stage (the TCGA cohort,

$267 \mathrm{p}=4 \mathrm{e}-05$; the GEO cohort, $\mathrm{p}=3.3 \mathrm{e}-06$; Figures S9D-E).

268

269

Functional analysis of circadian clock-based risk score

270

In the TCGA cohort, on the basis of the circadian clock-based risk score, 246 PC

271 patients were assigned to the high risk score group, while 247 PC patients were

272 assigned to the low risk score group. There were a total of 1114 DEGs between the

273 two groups, including 4643 upregulated and 6471 downregulated DEGs (Figure 8A). 
274 According to the GO analysis results, the top 3 enriched biological processes were 275 ribosome biogenesis (GO:0042254), viral gene expression (GO:0019080), and viral 276 transcription (GO:0019083; Figure 8B). For molecular function, the top 3 enriched 277 GO terms were small GTPase binding (GO:0031267), Ras GTPase binding 278 (GO:0017016), and structural constituent of ribosome (GO:0003735; Figure 8B). Moreover, Ribosome (hsa03010, p=5.15E-15) was also one of the top 10 circadian 280 clock-related pathways in PC (Figure 8C).

281

282

Correlation between CCMCCs and several key prognostic genes

283

By literature consulting, we noticed some key prognostic genes, such as PTEN, 284 TP53, BRCA1, BRCA2, ATM, RB1, PALB2, CHEK2, MLH1, MSH2, MSH6, and PMS2 $(34,37,45)$. Then, we explored the correlation between CCMCCs and the mentioned genes (Table S8 and Figure S10). We firstly evaluated the expression 287 levels of CCMCCs between different mutation status of key prognostic genes in PC 288 (Table S8). Importantly, in the TCGA cohort, the mutation rate of PTEN, TP53, BRCA1, BRCA2, ATM, RB1, PALB2, CHEK2, MLH1, MSH2, MSH6, and PMS2, was $21.26 \%, 15.99 \%, 2.23 \%, 5.06 \%, 6.07 \%, 9.72 \%, 1.62 \%, 1.62 \%, 0.81 \%, 0.61 \%$, $0.81 \%$, and $1.01 \%$, respectively. When compared to the PTEN wild-type group, higher expression of NR1D2 ( $\mathrm{p}=0.0008006)$, PRKAA1 $(\mathrm{p}=0.00005925)$, and RORB

$293(\mathrm{p}=0.00000054)$, were found in the PTEN mutation group (Table S8). When 294 compared to the TP53 wild-type group, higher expression of CRY1 $(\mathrm{p}=0.003774)$, 
295 CSNK1E ( $p=0.0004053)$, and RORB ( $p=0.006969)$, were found in the TP53 296 mutation group (Table S8). In PC, ATM mutation was correlated with high 297 expression levels of CLOCK $(\mathrm{p}=0.009059)$. Mutation status of other genes were also 298 related to CCMCCs' expression (Table S8).

299

300

Moreover, we investigated the correlation between the expression levels of 301 CCMCCs and the above important genes. As shown in Figure S10, a wild correlation 302 was found among these genes. It is worth mentioning that ATM expression had the highly positive relevance to CLOCK expression $(\mathrm{r}=0.633, \mathrm{p}<0.001)$. A highly positive correlation was also existed between RB1 and FBXL3 expression ( $\mathrm{r}=0.598$, 305 $\mathrm{p}<0.001$, Figure $\mathrm{S} 10)$

306

307

\section{Discussion}

308

Circadian clocks and circadian clock-related genes were essential to maintaining

309 homeostasis. Disruption of the circadian system and aberrant expression of

310 CCMCCs induced tumorigenesis and promoted the proliferation and invasion of

311 cancer cells (11-16). However, CCMCCs expression signature and its function in

312 PC have rarely been investigated. In the research, the expression profiles and 313 functions of CCMCCs were outlined. We also explored the close relationship 314 between CCMCCs and the prognosis of PC patients, thus developing a circadian 315 clock-based risk score model. The circadian clock-based risk score might participate 
316 in some biological processes and signaling pathways.

318 In the present study, we revealed the close relevance between the 22 enrolled 319 CCMCCs and prognosis. Cao et al. demonstrated that one of the core clock genes, 320 PER1, regulated the expression of androgen receptor, which might affect drug 321 sensitivity in PC (19). Additionally, in high-grade colon cancer, the relative low 322 expression of PER1 was found (22). In colon cancer and cholangiocarcinoma, the 323 overexpression of PER1 inhibited tumor progression and growth $(22,23)$. In PC, we 324 found a downregulation of PER1 in the T3-4 group in comparison with the T2 group, 325 which was consistent with its expression pattern in other cancer types. In our study, 326 we also detected overexpression of PER1 in the T2N0 stage. Moreover, the 327 expression level of PER1 was positively associated with OS in PC. In stomach 328 adenocarcinoma, patients with high FBXL3 expression showed poor clinical 329 outcome (24). However, in kidney renal clear cell carcinoma, an opposite result was 330 found. Specifically, Liu et al. found that patients with high FBXL3 expression 331 showed a better prognosis (24). In the 493 PC cases that enrolled in our study, high 332 FBXL3 expression was significantly correlated with longer DFS $(\mathrm{p}=0.012)$ and PFS $333(\mathrm{p}=0.0066)$. In breast cancer, overexpression of CSNK1D was found in the N1 group 334 (25). Similar overexpression trend of CSNK1D was also found in PC tissues with 335 N1 status. PC patients with short DFS ( $p=0.00032)$, PFS ( $\mathrm{p}=0.017)$, and OS $336(p=0.016)$ also showed overexpression of CSNK1D. In glioma, the expression level 
337 of BTRC was correlated with clinical outcome (26). Prognostic effects of some 338 CCMCCs in PC remain unclear. In PC, we found higher expression level of BTRC 339 in the T2N0 disease in comparison with the T3-4N1 disease. On the basis of $\mathrm{T}$ and $340 \mathrm{~N}$ stage, we divided the whole cohort and carried out the survival subgroup analysis. 341 As Tables S2-S7 shown, there were conflicting roles of some CCMCCs in the 342 prognosis of PC patients, such as DBP and NR1D1, suggesting different expression 343 patterns of CCMCCs in T2N0 and T3-4N1 disease. Collectively, in the study, we 344 fully investigated the association between 22 circadian clock-related genes and 345 clinical survival.

347 As one of the vital biomarkers in PC, high PSA levels also existed in some benign 348 diseases, such as prostatitis, prostatic hyperplasia, and after prostatic massage. Thus, 349 the clinical value of PSA in PC diagnosis and survival prediction remained 350 controversial (27-30). A multicenter trial reported that PSA failed to affect the 351 prognosis of PC (29), while another experiment found that PSA effectively reduced 352 the death rate of PC patients (30). In our study, the results showed that PSA level at 353 diagnosis was not statistically related to CCMCCs expression. Gleason grade was 354 another important biomarker in PC $(31,32)$. Recently, a meta-analysis found that 355 Gleason grade was positively associated with recurrence after surgery (33). Gleason 356 scoring system mainly concentrated on the pathological structure of PC, while the 357 transcriptomic and genomic features were missed. In the study, we attempted to find 
358 the correlation between Gleason score and gene expression. Nevertheless, a weak to

359 moderate correlation was found between Gleason score and expression levels of

CCMCCs. These results might demonstrate that the mechanisms of influences of

PSA level, Gleason score, and CCMCCs, on prognosis were different.

362

363

Not only clinical factors, but also lots of genes were considered as vitally prognostic

364 factors in PC, such as PTEN and TP53 (34-37). PTEN, one of the tumor suppressor genes, was commonly mutated in PC $(34,35,38)$. Several researches found that

PTEN mutation or down-expression had extensive influences on tumor microenvironment and PI3K signaling pathways, thus leading to tumor progression and poor prognosis in PC $(36,38-41)$. For instance, PTEN loss promoted T regulatory cell proliferation and infiltration, thereby promoting immune suppression and tumor metastasis (40). TP53 deficiency was presented in $10 \%$ to $40 \%$ of PC $(34,37)$. TP53 loss aggravated the genomic instability and activated several pathways, thus promoting tumor growth and poor outcome (42-44). In the enrolled cohort, we also found that the mutation rate of PTEN and TP53 was over $10 \%$, which was consistent with previous studies $(34-37,43,44)$. An increasing number of studies also highlighted the close relationship between clinical outcome and PTEN, TP53, BRCA1, BRCA2, ATM, RB1, PALB2, CHEK2, MLH1, MSH2, MSH6, and PMS2 $(34,37,45)$. However, few groups investigated the correlation between CCMCCs and the above prognostic genes. In our research, we considered 
379 the mutation status and expression levels of key genes. Through statistical analysis, 380 we found the link between CCMCCs and PTEN mutation, TP53 mutation, ATM 381 mutation, PTEN expression, TP53 expression, ATM expression, etc. These close 382 correlations might also explain why CCMCCs could be used for prognosis 383 prediction.

A certain number of existing prognostic models for PC patients were proposed (46, 386 47). These predictive models were developed by lncRNAs, miRNAs, or immune387 related genes $((46,47)$. Nevertheless, none of them involved circadian clock genes in. The mammalian circadian clock-related genes interacted with each other (48). CLOCK and ARNTL regulated the activity and expression of NR1D2, one of the 390 nuclear receptors. Subsequently, NR1D2 also could inhibit the mRNA level of ARNTL and NFIL3, leading to the repression of DBP. Collectively, extensive interactions of CCMCCs existed. It is important to make accurate predictions about the prognosis of PC patients for the development of precise treatment. In the present study, through COX regression analysis, we identified 9 vital CCMCCs which could predict prognosis in PC. In unselective PC patients who all received surgery, we developed the circadian clock-based risk score model with higher accuracy in the prediction of DFS than clinical features, including $\mathrm{T}$ stage and $\mathrm{N}$ stage. Then, we also verified the performance of the risk score model in T2N0 disease and T3-4N1 disease. Importantly, in DFS prediction, the risk score model showed preferable 
400

401 stage might be benefited more from the circadian clock-based risk score model. The 402 predictive model also performed well in terms of PFS and OS. The 9-CCMCCs 403 signature also reflected particular molecular functions, cellular components, 404 biological processes, and signaling pathways. Apart from clinical factors, such as T 405 stage, N stage, and PSA, the risk score model might put new insights in the prognosis 406 of PC from the aspect of circadian clock.

407

408

409

410

411

412

413

414

415

416

417 In summary, our study improved the understanding of the role of the circadian clock

418 in PC and proposed a circadian clock-based risk score model for prognostic

419 prediction. Moreover, PC patients at T2N0 stage might be benefited more from the

420 circadian clock-based risk score model. These results might be helpful for further 
421 investigations of the circadian clock - related molecular mechanisms and the 422 development of therapies for cancer.

423

424 Acknowledges

425 Consent for publication: All participants gave their informed consent for 426 publication.

427 Conflicts and interests: There were no conflicts and interests.

428 Authors' contributions : (I) Conception and design: Wenchang Yue, Xiao Du; (II) 429 Administrative support: Jianquan Hou; (III) Collection and assembly of data: 430 Wenchang Yue, Xiao Du; (IV) Data analysis and interpretation: Wenchang Yue, 431 Xiao Du; (V) Manuscript writing: All authors; (VI) Final approval of manuscript: 432 All authors

433

434 References

435 1. Siegel RL, Miller KD, Fuchs HE, Jemal A. Cancer Statistics, 2021. CA: a cancer 436 journal for clinicians. 2021;71(1):7-33.

437 2. Chen $\mathrm{W}$, Zheng R, Baade PD, Zhang S, Zeng H, Bray F, Jemal A, Yu XQ, He J. 438 Cancer statistics in China, 2015. CA: a cancer journal for clinicians. 2016;66(2):11543932.

440 3. Jemal A, Ma J, Siegel R, Fedewa S, Brawley O, Ward EM. Prostate Cancer 441 Incidence Rates 2 Years After the US Preventive Services Task Force 
442 Recommendations Against Screening. JAMA oncology. 2016;2(12):1657-60.

443 4. Seikkula HA, Kaipia AJ, Ryynänen H, Seppä K, Pitkäniemi JM, Malila NK, 444 Boström PJ. The impact of socioeconomic status on stage specific prostate cancer 445 survival and mortality before and after introduction of PSA test in Finland. 446 International journal of cancer. 2018;142(5):891-8.

447 5. Small EJ, Saad F, Chowdhury S, Oudard S, Hadaschik BA, Graff JN, Olmos D, 448 Mainwaring PN, Lee JY, Uemura H, De Porre P, Smith AA, Zhang K, Lopez-Gitlitz 449 A, Smith MR. Apalutamide and overall survival in non-metastatic castration450 resistant prostate cancer. Annals of oncology : official journal of the European 451 Society for Medical Oncology. 2019;30(11):1813-20.

452 6. Saad F, Bögemann M, Suzuki K, Shore N. Treatment of nonmetastatic 453 castration-resistant prostate cancer: focus on second-generation androgen receptor 454 inhibitors. Prostate cancer and prostatic diseases. 2021.

455 7. Callaway E, Ledford H. Medicine Nobel awarded for work on circadian clocks. $456 \quad$ Nature. 2017;550(7674):18.

457 8. Burki T. Nobel Prize awarded for discoveries in circadian rhythm. Lancet 458 (London, England). 2017;390(10104):e25.

459 9. Sigurdardottir LG, Valdimarsdottir UA, Fall K, Rider JR, Lockley SW, 460 Schernhammer E, Mucci LA. Circadian disruption, sleep loss, and prostate cancer 461 risk: a systematic review of epidemiologic studies. Cancer epidemiology, 462 biomarkers \& prevention : a publication of the American Association for Cancer 
463 Research, cosponsored by the American Society of Preventive Oncology. $464 \quad 2012 ; 21(7): 1002-11$.

465 10. Wendeu-Foyet MG, Menegaux F. Circadian Disruption and Prostate Cancer 466 Risk: An Updated Review of Epidemiological Evidences. Cancer epidemiology, 467 biomarkers \& prevention : a publication of the American Association for Cancer 468 Research, cosponsored by the American Society of Preventive Oncology. $469 \quad 2017 ; 26(7): 985-91$.

470 11. Viswanathan AN, Hankinson SE, Schernhammer ES. Night shift work and the 471 risk of endometrial cancer. Cancer research. 2007;67(21):10618-22.

472 12. Stevens RG, Brainard GC, Blask DE, Lockley SW, Motta ME. Breast cancer and 473 circadian disruption from electric lighting in the modern world. CA: a cancer journal 474 for clinicians. 2014;64(3):207-18.

475 13. Papagiannakopoulos T, Bauer MR, Davidson SM, Heimann M, Subbaraj L, 476 Bhutkar A, Bartlebaugh J, Vander Heiden MG, Jacks T. Circadian Rhythm 477 Disruption Promotes Lung Tumorigenesis. Cell metabolism. 2016;24(2):324-31. 478 14. Kettner NM, Voicu H, Finegold MJ, Coarfa C, Sreekumar A, Putluri N, Katchy 479 CA, Lee C, Moore DD, Fu L. Circadian Homeostasis of Liver Metabolism 480 Suppresses Hepatocarcinogenesis. Cancer cell. 2016;30(6):909-24.

481 15. Innominato PF, Focan C, Gorlia T, Moreau T, Garufi C, Waterhouse J, 482 Giacchetti S, Coudert B, Iacobelli S, Genet D, Tampellini M, Chollet P, Lentz MA, 
483 Mormont MC, Lévi F, Bjarnason GA. Circadian rhythm in rest and activity: a 484 biological correlate of quality of life and a predictor of survival in patients with 485 metastatic colorectal cancer. Cancer research. 2009;69(11):4700-7.

486 16. Huisman SA, Oklejewicz M, Ahmadi AR, Tamanini F, Ijzermans JN, van der 487 Horst GT, de Bruin RW. Colorectal liver metastases with a disrupted circadian 488 rhythm phase shift the peripheral clock in liver and kidney. International journal of 489 cancer. 2015;136(5):1024-32. 17. Takahashi JS. Transcriptional architecture of the mammalian circadian clock.

491 Nature reviews Genetics. 2017;18(3):164-79.

18. Partch CL, Green CB, Takahashi JS. Molecular architecture of the mammalian 493 circadian clock. Trends in cell biology. 2014;24(2):90-9.

19. Cao Q, Gery S, Dashti A, Yin D, Zhou Y, Gu J, Koeffler HP. A role for the clock 495 gene per1 in prostate cancer. Cancer research. 2009;69(19):7619-25.

496 20. Ross-Adams H, Lamb AD, Dunning MJ, Halim S, Lindberg J, Massie CM, 497 Egevad LA, Russell R, Ramos-Montoya A, Vowler SL, Sharma NL, Kay J, 498 Whitaker H, Clark J, Hurst R, Gnanapragasam VJ, Shah NC, Warren AY, Cooper CS, Lynch AG, Stark R, Mills IG, Grönberg H, Neal DE. Integration of copy number and transcriptomics provides risk stratification in prostate cancer: A discovery and 501 validation cohort study. EBioMedicine. 2015;2(9):1133-44.

502 21. Liu J, Lichtenberg T, Hoadley KA, Poisson LM, Lazar AJ, Cherniack AD, 503 Kovatich AJ, Benz CC, Levine DA, Lee AV, Omberg L, Wolf DM, Shriver CD, 
504 Thorsson V, Hu H. An Integrated TCGA Pan-Cancer Clinical Data Resource to 505 Drive High-Quality Survival Outcome Analytics. Cell. 2018;173(2):400-16.e11.

506 22. Krugluger W, Brandstaetter A, Kállay E, Schueller J, Krexner E, Kriwanek S, 507 Bonner E, Cross HS. Regulation of genes of the circadian clock in human colon 508 cancer: reduced period-1 and dihydropyrimidine dehydrogenase transcription 509 correlates in high-grade tumors. Cancer research. 2007;67(16):7917-22.

510 23. Han Y, Meng F, Venter J, Wu N, Wan Y, Standeford H, Francis H, Meininger 511 C, Greene J, Jr., Trzeciakowski JP, Ehrlich L, Glaser S, Alpini G. miR-34a512 dependent overexpression of Per1 decreases cholangiocarcinoma growth. Journal of 513 hepatology. 2016;64(6):1295-304.

514 24. Liu Z, Yu K, Zheng J, Lin H, Zhao Q, Zhang X, Feng W, Wang L, Xu J, Xie D, 515 Zuo ZX, Liu ZX, Zheng Q. Dysregulation, functional implications, and prognostic 516 ability of the circadian clock across cancers. Cancer medicine. 2019;8(4):1710-20. 517 25. Abba MC, Sun H, Hawkins KA, Drake JA, Hu Y, Nunez MI, Gaddis S, Shi T, 518 Horvath S, Sahin A, Aldaz CM. Breast cancer molecular signatures as determined 519 by SAGE: correlation with lymph node status. Molecular cancer research : MCR. $520 \quad 2007 ; 5(9): 881-90$.

521 26. Zhou DD, Li HL, Liu W, Zhang LP, Zheng Q, Bai J, Hu YQ, Yin CG, Lv SJ, 522 Zhang BG. miR-193a-3p Promotes the Invasion, Migration, and Mesenchymal 523 Transition in Glioma through Regulating BTRC. BioMed research international. 524 2021;2021:8928509. 
525 27. Draisma G, Boer R, Otto SJ, van der Cruijsen IW, Damhuis RA, Schröder FH, 526 de Koning HJ. Lead times and overdetection due to prostate-specific antigen 527 screening: estimates from the European Randomized Study of Screening for Prostate 528 Cancer. Journal of the National Cancer Institute. 2003;95(12):868-78.

529 28. Manceau C, Fromont G, Beauval JB, Barret E, Brureau L, Créhange G, Dariane 530 C, Fiard G, Gauthé M, Mathieu R, Renard-Penna R, Roubaud G, Ruffion A, Sargos 531 P, Rouprêt M, Ploussard G, On Behalf Of The Cc-Afu Cancerology Committee Of 532 The Association Française dU. Biomarker in Active Surveillance for Prostate 533 Cancer: A Systematic Review. Cancers. 2021;13(17).

534 29. Andriole GL, Crawford ED, Grubb RL, 3rd, Buys SS, Chia D, Church TR, Fouad 535 MN, Isaacs C, Kvale PA, Reding DJ, Weissfeld JL, Yokochi LA, O'Brien B, Ragard 536 LR, Clapp JD, Rathmell JM, Riley TL, Hsing AW, Izmirlian G, Pinsky PF, Kramer 537 BS, Miller AB, Gohagan JK, Prorok PC. Prostate cancer screening in the randomized 538 Prostate, Lung, Colorectal, and Ovarian Cancer Screening Trial: mortality results 539 after 13 years of follow-up. Journal of the National Cancer Institute. $540 \quad 2012 ; 104(2): 125-32$.

541 30. Schröder FH, Hugosson J, Roobol MJ, Tammela TL, Ciatto S, Nelen V, 542 Kwiatkowski M, Lujan M, Lilja H, Zappa M, Denis LJ, Recker F, Páez A, Määttänen 543 L, Bangma CH, Aus G, Carlsson S, Villers A, Rebillard X, van der Kwast T, Kujala 544 PM, Blijenberg BG, Stenman UH, Huber A, Taari K, Hakama M, Moss SM, de 545 Koning HJ, Auvinen A. Prostate-cancer mortality at 11 years of follow-up. The New 
546 England journal of medicine. 2012;366(11):981-90.

547 31. Sopyllo K, Erickson AM, Mirtti T. Grading Evolution and Contemporary 548 Prognostic Biomarkers of Clinically Significant Prostate Cancer. Cancers. $549 \quad 2021 ; 13(4)$.

550 32. Moris L, Cumberbatch MG, Van den Broeck T, Gandaglia G, Fossati N, Kelly 551 B, Pal R, Briers E, Cornford P, De Santis M, Fanti S, Gillessen S, Grummet JP, 552 Henry AM, Lam TBL, Lardas M, Liew M, Mason MD, Omar MI, Rouvière O, 553 Schoots IG, Tilki D, van den Bergh RCN, van Der Kwast TH, van Der Poel HG, 554 Willemse PM, Yuan CY, Konety B, Dorff T, Jain S, Mottet N, Wiegel T. Benefits 555 and Risks of Primary Treatments for High-risk Localized and Locally Advanced 556 Prostate Cancer: An International Multidisciplinary Systematic Review. European 557 urology. 2020;77(5):614-27.

558 33. John A, John H, Catterwell R, Selth LA, Callaghan MO. Primary Gleason grade 559 and Gleason grade group at positive surgical margins: a systematic review and meta560 analysis. BJU international. 2021;127 Suppl 1:13-22.

561 34. Rebello RJ, Oing C, Knudsen KE, Loeb S, Johnson DC, Reiter RE, Gillessen S, 562 Van der Kwast T, Bristow RG. Prostate cancer. Nature reviews Disease primers. $563 \quad 2021 ; 7(1): 9$.

564 35. Vitkin N, Nersesian S, Siemens DR, Koti M. The Tumor Immune Contexture of 565 Prostate Cancer. Frontiers in immunology. 2019;10:603.

566 36. Vidotto T, Melo CM, Castelli E, Koti M, Dos Reis RB, Squire JA. Emerging 
567 role of PTEN loss in evasion of the immune response to tumours. British journal of 568 cancer. 2020;122(12):1732-43.

569 37. Muñoz-Fontela C, Mandinova A, Aaronson SA, Lee SW. Emerging roles of p53 570 and other tumour-suppressor genes in immune regulation. Nature reviews 571 Immunology. 2016;16(12):741-50.

572 38. Jamaspishvili T, Berman DM, Ross AE, Scher HI, De Marzo AM, Squire JA, 573 Lotan TL. Clinical implications of PTEN loss in prostate cancer. Nature reviews 574 Urology. 2018;15(4):222-34.

575 39. Garcia AJ, Ruscetti M, Arenzana TL, Tran LM, Bianci-Frias D, Sybert E, 576 Priceman SJ, Wu L, Nelson PS, Smale ST, Wu H. Pten null prostate epithelium 577 promotes localized myeloid-derived suppressor cell expansion and immune 578 suppression during tumor initiation and progression. Molecular and cellular biology. $579 \quad 2014 ; 34(11): 2017-28$.

580 40. Vidotto T, Saggioro FP, Jamaspishvili T, Chesca DL, Picanço de Albuquerque 581 CG, Reis RB, Graham CH, Berman DM, Siemens DR, Squire JA, Koti M. PTEN582 deficient prostate cancer is associated with an immunosuppressive tumor 583 microenvironment mediated by increased expression of IDO1 and infiltrating 584 FoxP3+ T regulatory cells. 2019;79(9):969-79.

585 41. Toso A, Revandkar A, Di Mitri D, Guccini I, Proietti M, Sarti M, Pinton S, 586 Zhang J, Kalathur M, Civenni G, Jarrossay D, Montani E, Marini C, Garcia587 Escudero R, Scanziani E, Grassi F, Pandolfi PP, Catapano CV, Alimonti A. 
588 Enhancing chemotherapy efficacy in Pten-deficient prostate tumors by activating the 589 senescence-associated antitumor immunity. Cell reports. 2014;9(1):75-89.

590 42. Bezzi M, Seitzer N, Ishikawa T, Reschke M, Chen M, Wang G, Mitchell C, Ng

591 C, Katon J, Lunardi A, Signoretti S, Clohessy JG, Zhang J, Pandolfi PP. Diverse 592 genetic-driven immune landscapes dictate tumor progression through distinct 593 mechanisms. Nature medicine. 2018;24(2):165-75.

594 43. Robinson D, Van Allen EM, Wu YM, Schultz N, Lonigro RJ, Mosquera JM, 595 Montgomery B, Taplin ME, Pritchard CC, Attard G, Beltran H, Abida W, Bradley 596 RK, Vinson J, Cao X, Vats P, Kunju LP, Hussain M, Feng FY, Tomlins SA, Cooney 597 KA, Smith DC, Brennan C, Siddiqui J, Mehra R, Chen Y, Rathkopf DE, Morris MJ, 598 Solomon SB, Durack JC, Reuter VE, Gopalan A, Gao J, Loda M, Lis RT, Bowden 599 M, Balk SP, Gaviola G, Sougnez C, Gupta M, Yu EY, Mostaghel EA, Cheng HH, 600 Mulcahy H, True LD, Plymate SR, Dvinge H, Ferraldeschi R, Flohr P, Miranda S, 601 Zafeiriou Z, Tunariu N, Mateo J, Perez-Lopez R, Demichelis F, Robinson BD, 602 Schiffman M, Nanus DM, Tagawa ST, Sigaras A, Eng KW, Elemento O, Sboner A, 603 Heath EI, Scher HI, Pienta KJ, Kantoff P, de Bono JS, Rubin MA, Nelson PS, 604 Garraway LA, Sawyers CL, Chinnaiyan AM. Integrative clinical genomics of 605 advanced prostate cancer. Cell. 2015;161(5):1215-28. 44. Hamid AA, Gray KP, Shaw G, MacConaill LE, Evan C, Bernard B, Loda M, 607 Corcoran NM, Van Allen EM, Choudhury AD, Sweeney CJ. Compound Genomic 608 Alterations of TP53, PTEN, and RB1 Tumor Suppressors in Localized and 
609 Metastatic Prostate Cancer. European urology. 2019;76(1):89-97.

610 45. Abida W, Cyrta J, Heller G, Prandi D, Armenia J, Coleman I, Cieslik M, Benelli

611 M, Robinson D, Van Allen EM, Sboner A, Fedrizzi T, Mosquera JM, Robinson BD,

612 De Sarkar N, Kunju LP, Tomlins S, Wu YM, Nava Rodrigues D, Loda M, Gopalan

613 A, Reuter VE, Pritchard CC, Mateo J, Bianchini D, Miranda S, Carreira S, Rescigno

614 P, Filipenko J, Vinson J, Montgomery RB, Beltran H, Heath EI, Scher HI, Kantoff

615 PW, Taplin ME, Schultz N, deBono JS, Demichelis F, Nelson PS, Rubin MA,

616 Chinnaiyan AM, Sawyers CL. Genomic correlates of clinical outcome in advanced

617 prostate cancer. Proceedings of the National Academy of Sciences of the United

618 States of America. 2019;116(23):11428-36.

619 46. Zhang E, He J, Zhang H, Shan L, Wu H, Zhang M, Song Y. Immune-Related

620 Gene-Based Novel Subtypes to Establish a Model Predicting the Risk of Prostate

621 Cancer. Frontiers in genetics. 2020;11:595657.

622 47. Xiaoli Z, Yawei W, Lianna L, Haifeng L, Hui Z. Screening of Target Genes and

623 Regulatory Function of miRNAs as Prognostic Indicators for Prostate Cancer.

624 Medical science monitor : international medical journal of experimental and clinical

625 research. 2015;21:3748-59.

626 48. Lehmann R, Childs L, Thomas P, Abreu M, Fuhr L, Herzel H, Leser U, Relógio

627 A. Assembly of a comprehensive regulatory network for the mammalian circadian

628 clock: a bioinformatics approach. PloS one. 2015;10(5):e0126283. 
Figure 1

Figure 1. Expression levels of the core components of the mammalian circadian clock (CCMCCs) in prostate cancer and its correlation with clinical factors.

A) The expression profile of CCMCCs in prostate cancer. B) The expression level of SKP1 was significantly associated with age $(p=0.0079)$. C) Seven CCMCCs differentially expressed between the N0 group and the N1 group (p<0.05). D) Ten CCMCCs differentially expressed between the T2 group and the T3-4 group $(p<0.05)$. Abbreviation: $N$, lymph node; T, tumor.
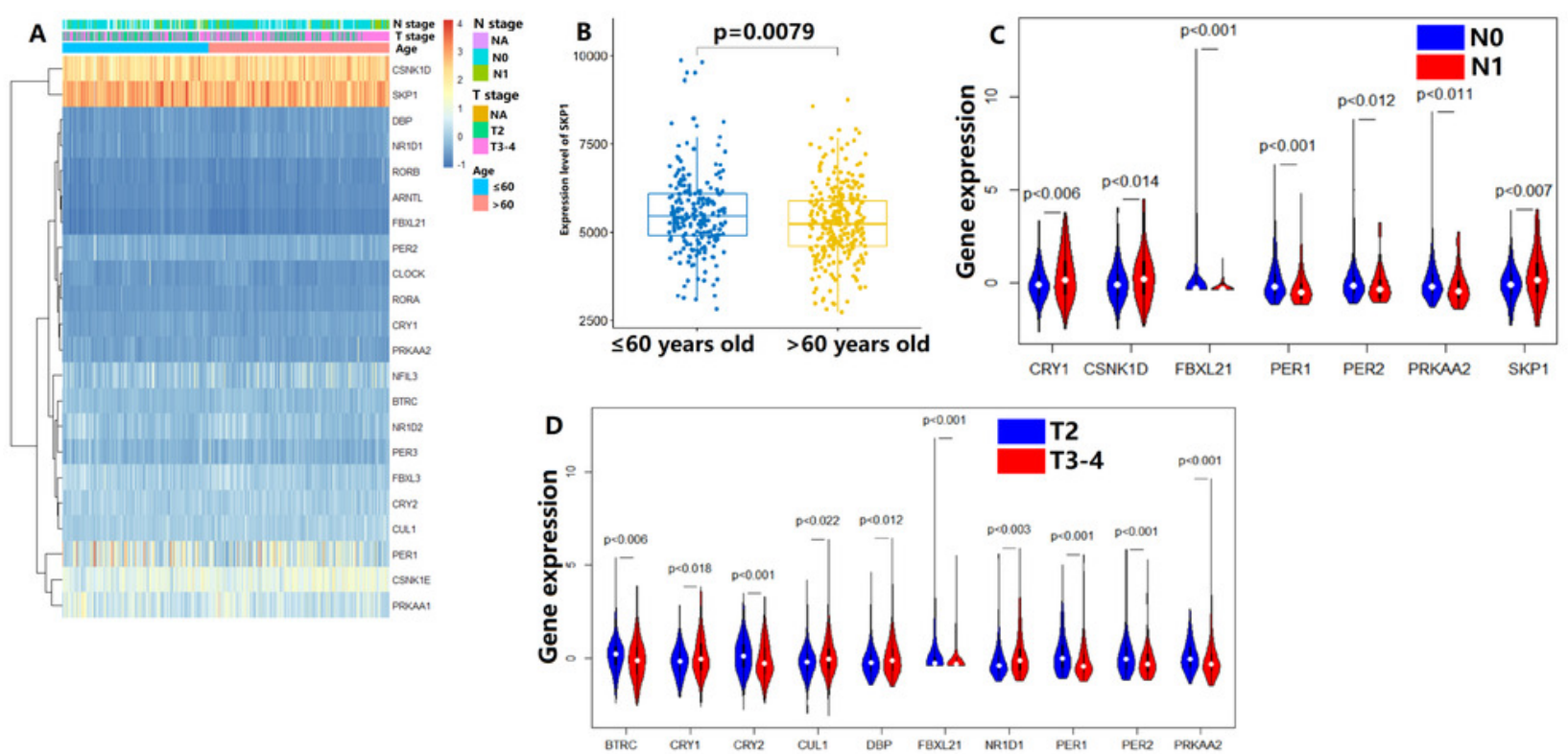
Figure 2

Figure 2. The expression levels of five core clock genes were related to disease-free survival (DFS).

A) ARNTL $(p=0.037) ; B) C L O C K(p=0.006) ; C) C R Y 1(p=0.0015) ; D)$ PER2 $(p=0.0051) ;$ E)PER3 $(p=0.013)$.
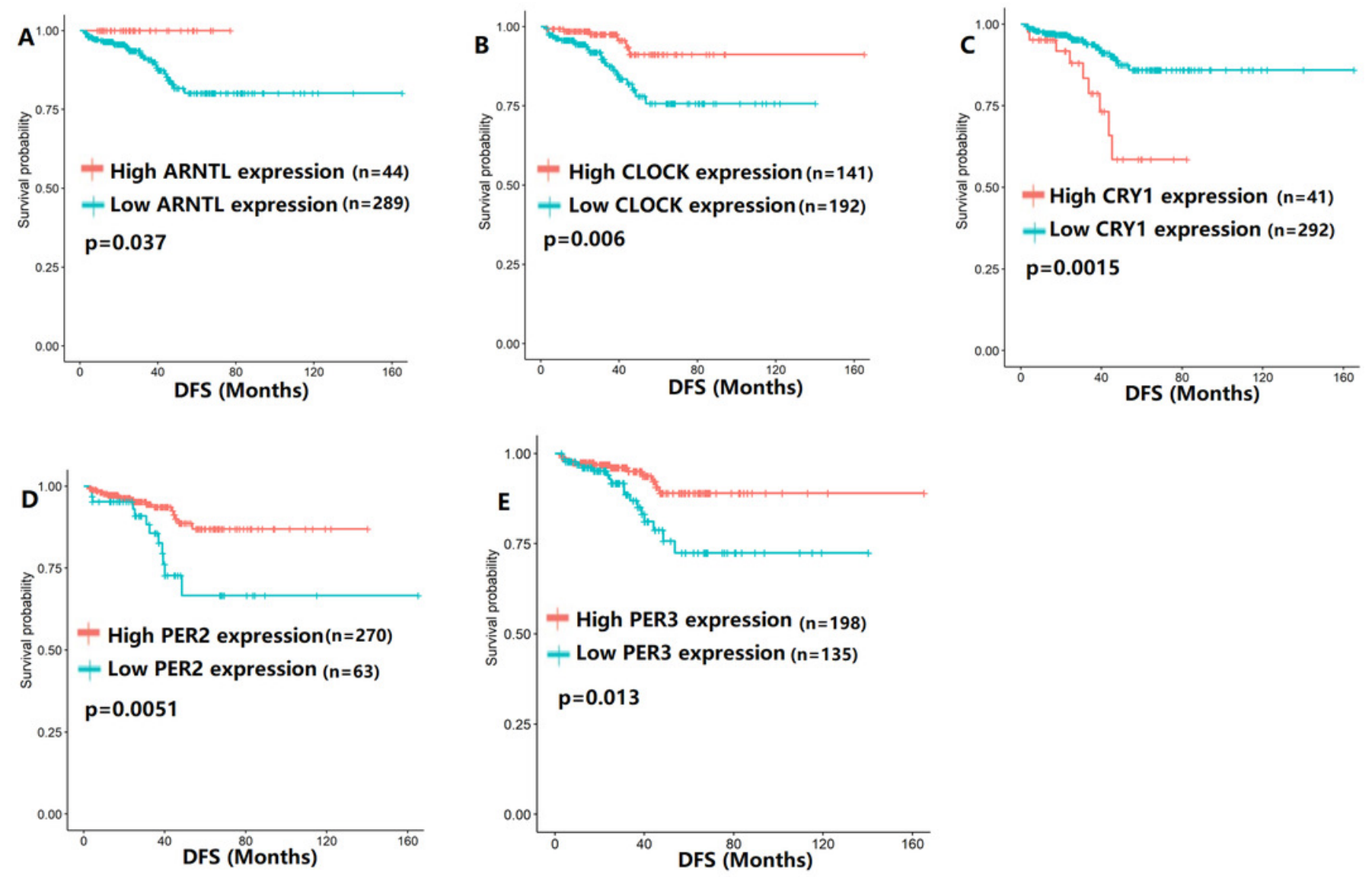
Figure 3

Figure 3. The expression levels of 12 circadian clock-related genes were related to disease-free survival (DFS).
A)BTRC $(p=0.026) ; B) \operatorname{CSNK1D}(p=0.00032) ; C) \operatorname{CSNK1E}(p=0.0019) ; D) C U L 1(p=0.0054) ;$
E)DBP ( $p=0.00075) ;$ F)FBXL21 ( $p=0.00035) ; G) F B X L 3(p=0.012) ;$ H)NR1D1 $(p=0.04)$;
I)PRKAA1 ( $p=0.013) ; j)$ PRKAA2 $(p=0.015) ; \operatorname{K}) \operatorname{RORB}(p=0.0067) ;$ L) SKP1 $(p=0.017)$.
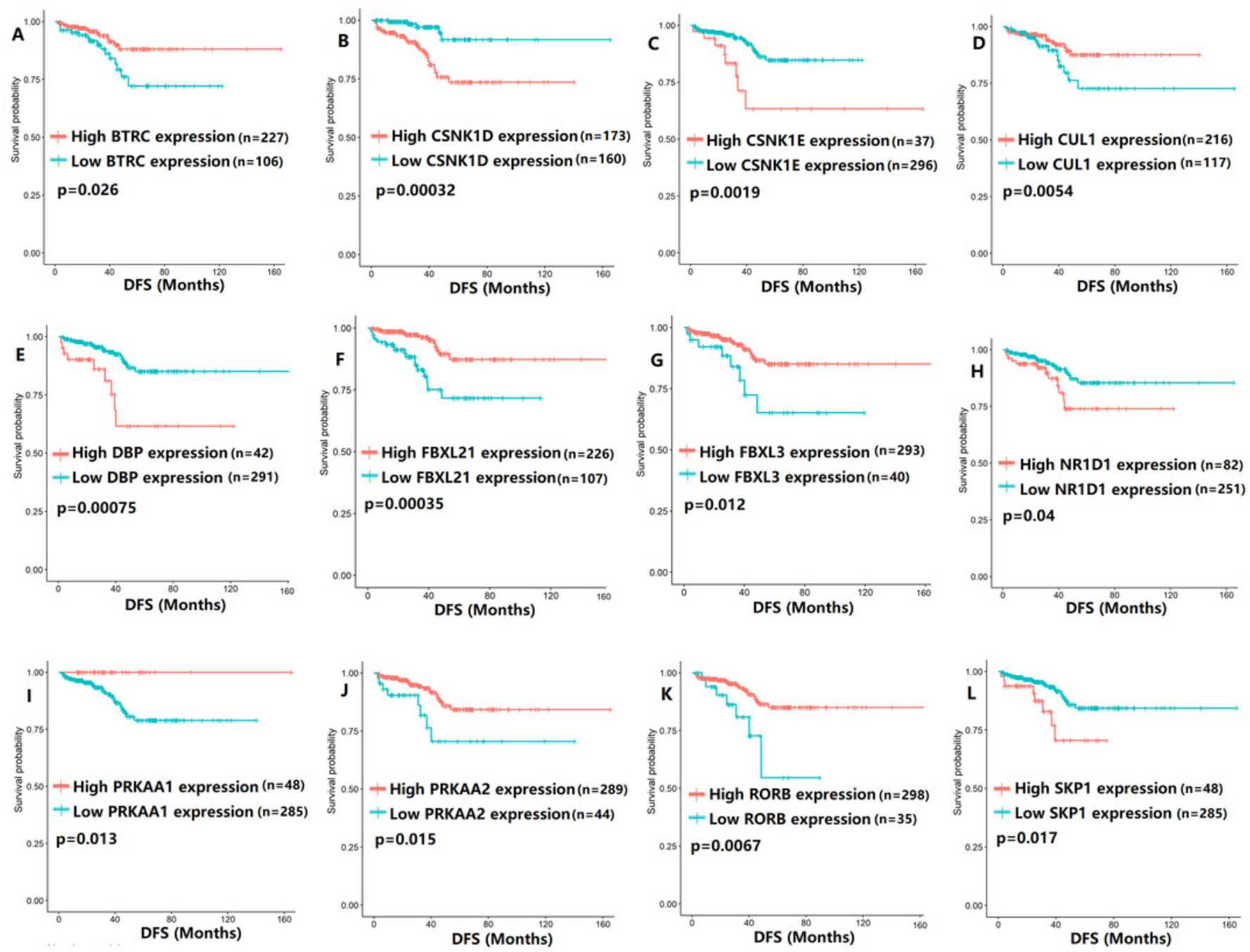
Figure 4

Figure 4. The expression levels of four core clock genes were related to progression-free survival (PFS).

A) CLOCK $(p=0.0085) ; B) C R Y 1(p=0.0028) ; C)$ PER2 $(p=0.032) ; D)$ PER3 $(p=0.045)$.
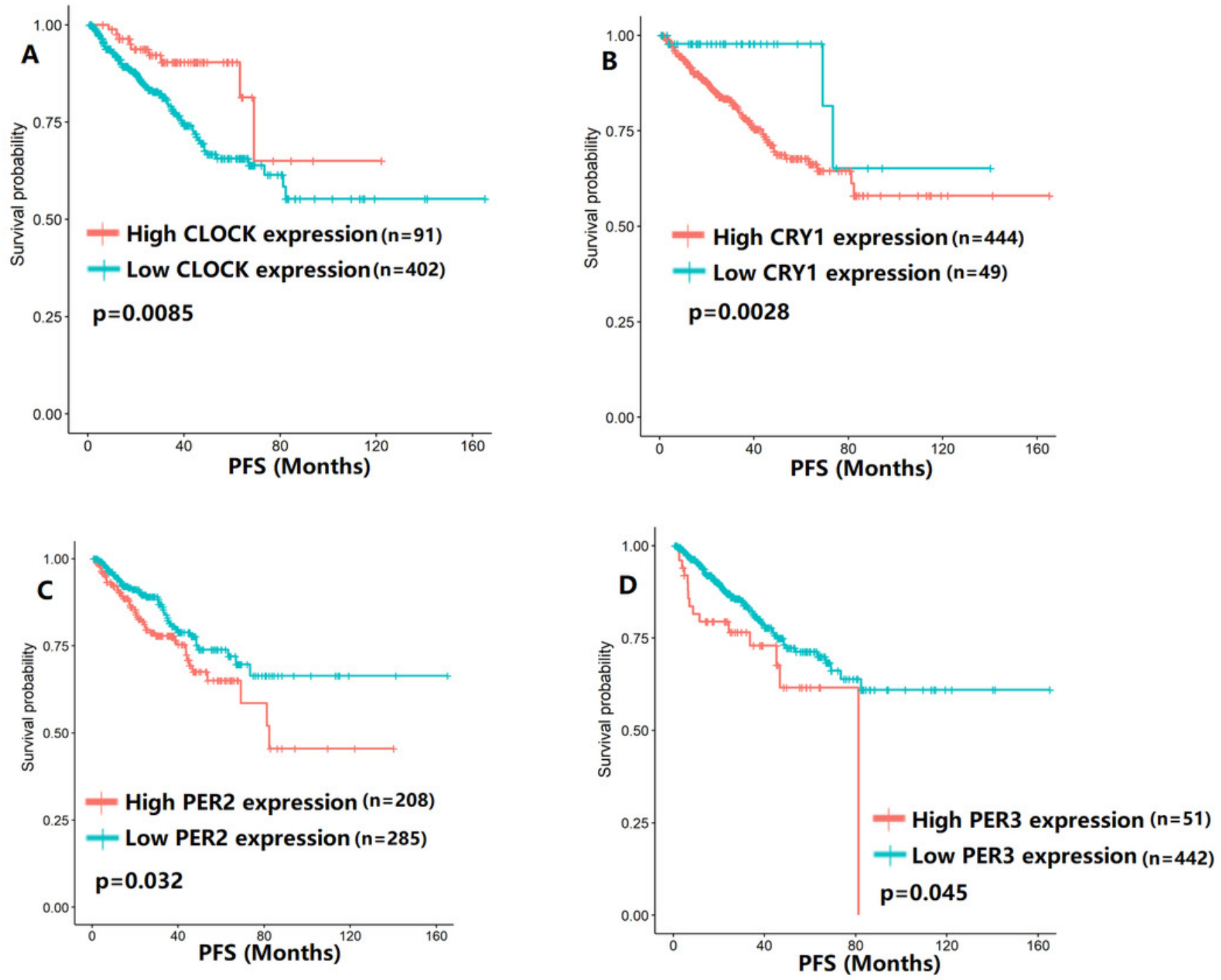
Figure 5

Figure 5. The expression levels of 14 circadian clock-related genes were related to progression-free survival (PFS).

A) BTRC $(p=0.031) ; \operatorname{B}) \operatorname{CSNK1D}(p=0.017) ; \operatorname{C}) \operatorname{CSNK1E}(p=0.00026) ; D) C U L 1(p=0.025) ; E)$ DBP $(p=0.00092) ;$ F)FBXL21 ( $p=0.0014) ; G)$ FBXL3 $(p=0.0066) ;$ H)NFIL3 $(p=0.035) ;$ I)NR1D1 $(p=0.027) ; J) N R 1 D 2(p=0.044) ;$ K)PRKAA1 $(p=0.023) ;$ L)PRKAA2 $(p=0.0051) ; M) R O R B$ $(p<0.0001) ; N)$ SKP1 $(p=0.014)$.
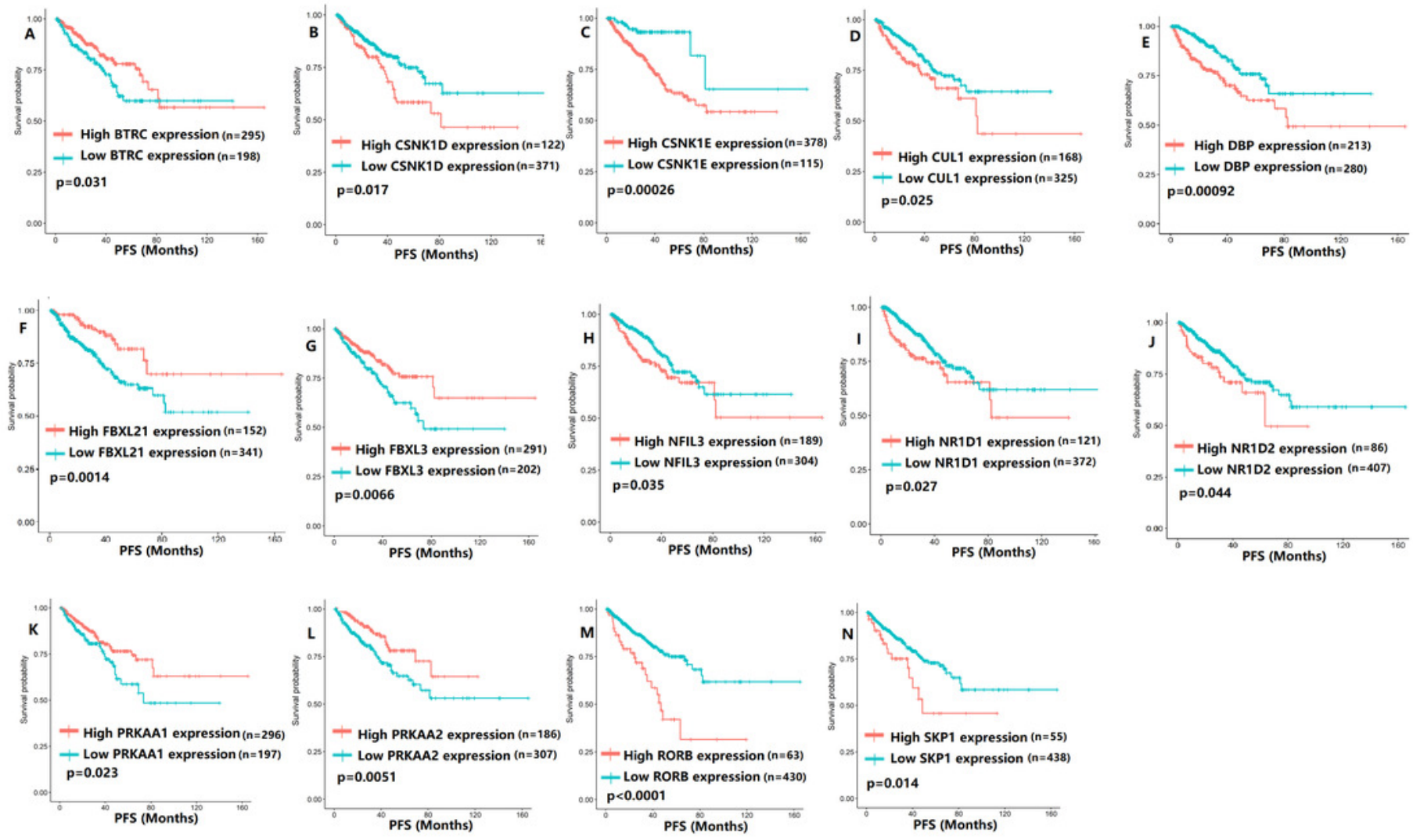
Figure 6

Figure 6. The expression levels of five the core components of the mammalian circadian clock were related to overall survival (OS).

A) CSNK1D ( $p=0.016) ; B) F B X L 21(p=0.039) ; C) N F I L 3(p=0.037) ; D)$ PER1 $(p=0.035) ;$ E)RORB $(p=0.037)$.
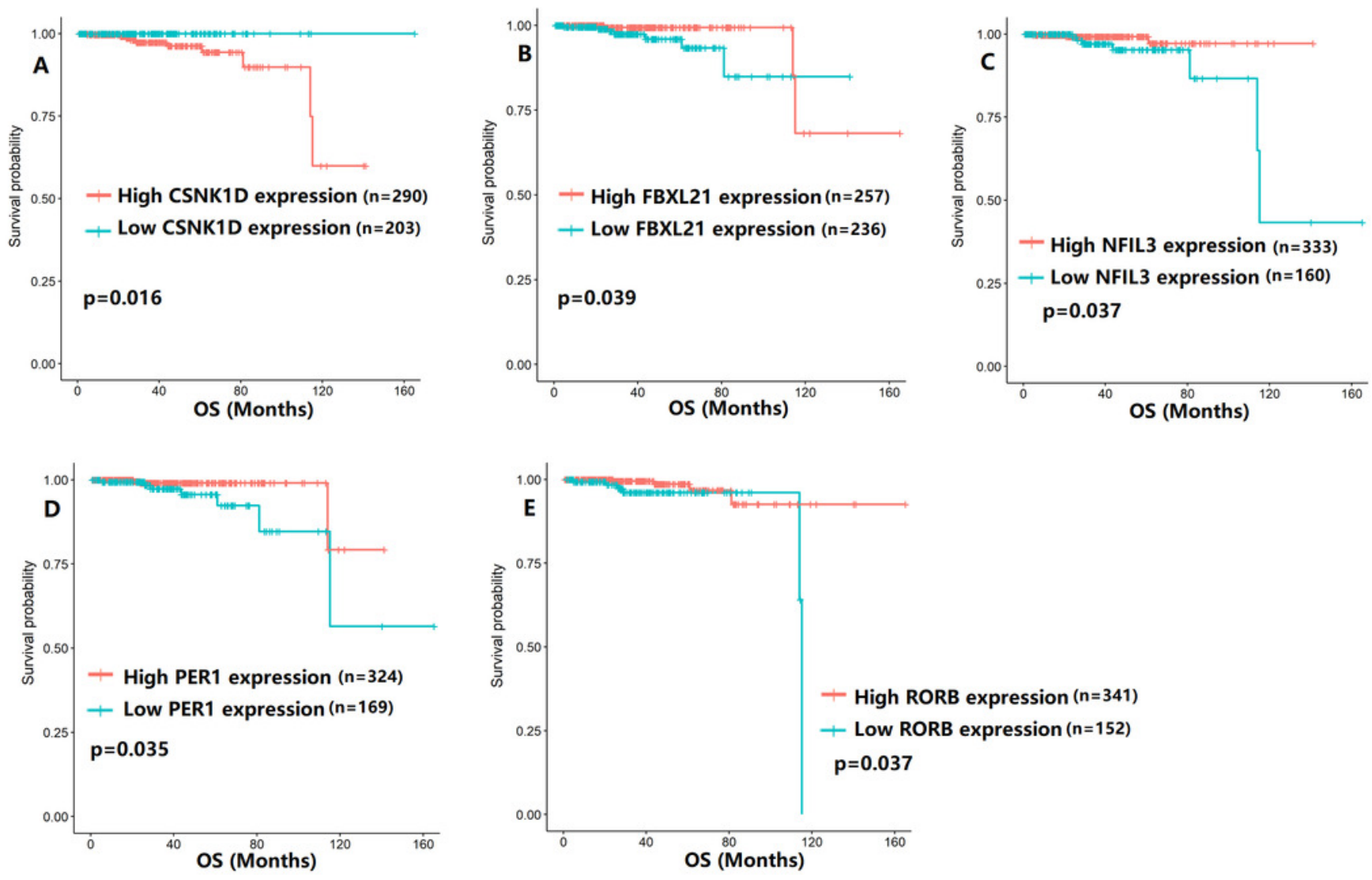


\section{Figure 7}

Figure 7. Validation of proposed circadian clock-based risk score model in disease-free survival (DFS) prediction by receiver-operator characteristic (ROC) analyses.

$A-B)$ ROC curves in the training cohort $(A U C=0.742)$ and the validation cohort $(A U C=0.821)$ for 3-year. C-D)ROC curves in the training cohort $(A U C=0.728)$ and the validation cohort $(A U C=0.753)$ for 5-year. E) High circadian clock-based risk score was correlated with shorter DFS $(p<0.0001)$.

\section{3-year ROC}
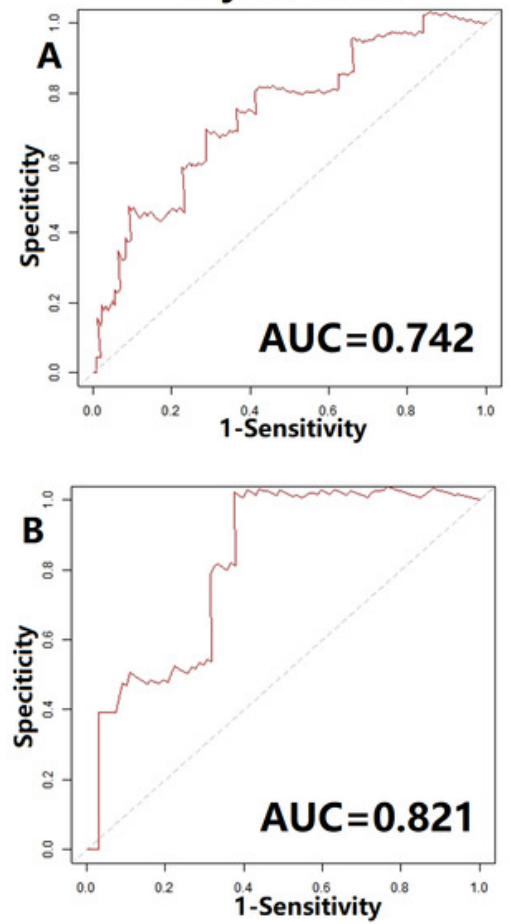

5-year ROC
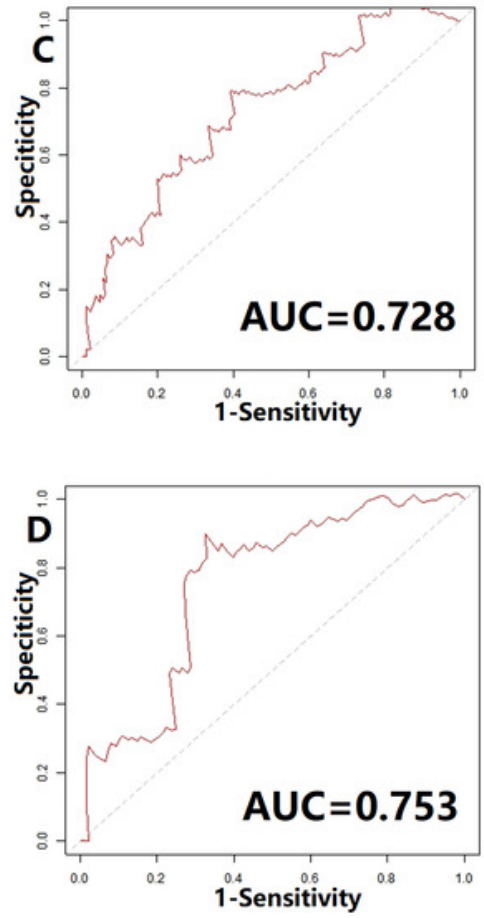

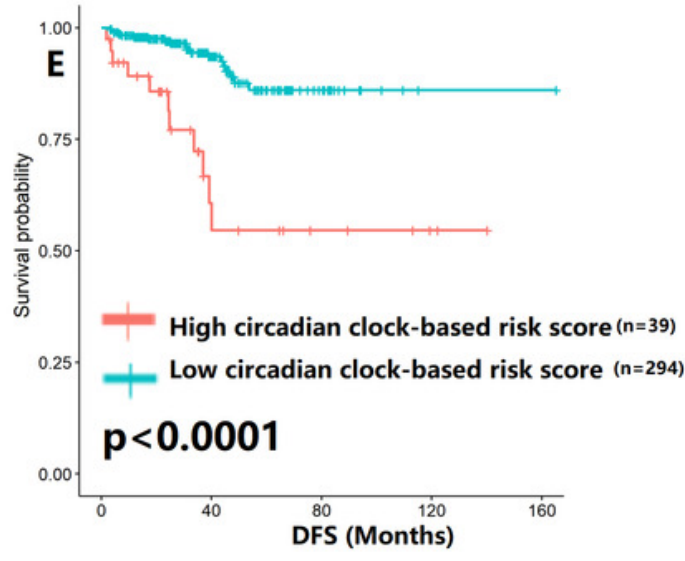


Figure 8

Figure 8. Functional enrichment analysis of circadian clock-based risk score.

A) The volcano plot visualized a total of 11114 differentially expressed genes (DEGs) were found between the high and low circadian clock-based risk score groups $(p<0.05)$. B) The top 10 circadian clock-related Gene Ontology(GO) terms. C). The top 10 circadian clock-related pathways were found by the the Kyoto Encyclopedia of Genes and Genomes (KEGG) enrichment analyses.
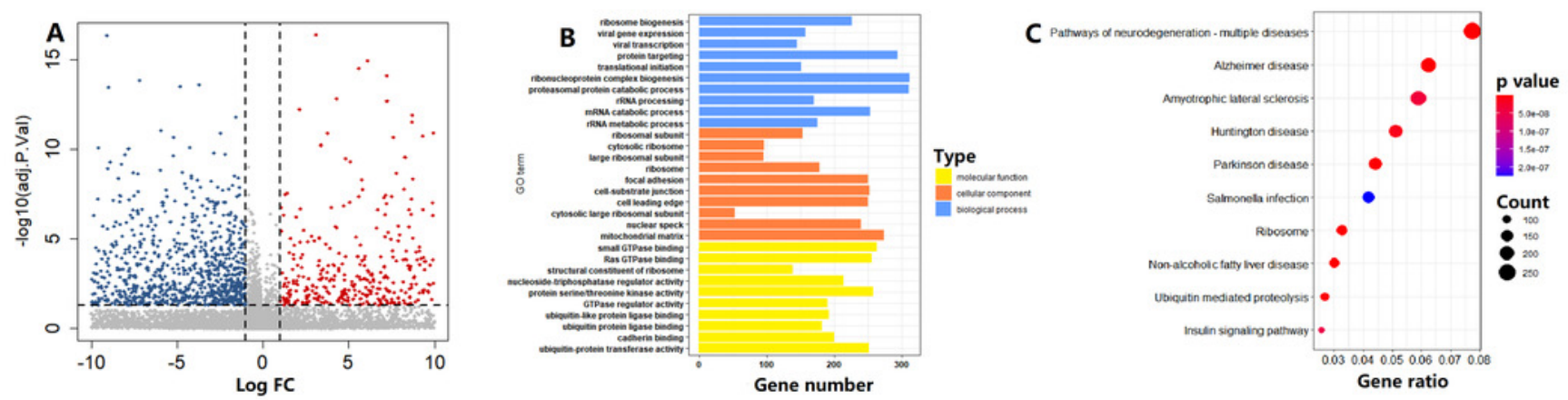


\section{Table 1 (on next page)}

Table1 Cox regression analysis for disease-free survival 
1 Table1 Cox regression analysis for disease-free survival

\begin{tabular}{|c|c|c|c|c|}
\hline Variables & HR & Lower limit of $95 \%$ CI & Upper limit of $95 \%$ CI & $\mathbf{p}$ \\
\hline ARNTL & 0.996443 & 0.989075 & 1.003865 & 0.34664 \\
\hline BTRC & 0.998178 & 0.996644 & 0.999715 & $\underline{0.020136}$ \\
\hline CLOCK & 0.997669 & 0.995476 & 0.999866 & $\underline{0.037606}$ \\
\hline CRY1 & 1.001943 & 0.999512 & 1.004381 & 0.117348 \\
\hline CRY2 & 1.000162 & 0.999264 & 1.001061 & 0.723401 \\
\hline CSNK1D & 1.000571 & 1.000203 & 1.000939 & $\underline{0.002343}$ \\
\hline CSNK1E & 1.00061 & 1.000175 & 1.001045 & $\underline{0.006002}$ \\
\hline CUL1 & 1.00047 & 0.998849 & 1.002094 & 0.569861 \\
\hline DBP & 1.001227 & 0.999326 & 1.003132 & 0.205956 \\
\hline FBXL21 & 0.991817 & 0.942722 & 1.043469 & 0.751078 \\
\hline FBXL3 & 0.998762 & 0.997736 & 0.999788 & $\underline{0.01807}$ \\
\hline NFIL3 & 1.000172 & 0.999762 & 1.000582 & 0.411733 \\
\hline NR1D1 & 1.00041 & 0.998809 & 1.002014 & 0.615872 \\
\hline NR1D2 & 0.999767 & 0.998967 & 1.000568 & 0.56873 \\
\hline PER1 & 0.999872 & 0.99963 & 1.000115 & 0.301755 \\
\hline PER2 & 0.999433 & 0.99829 & 1.000576 & 0.330692 \\
\hline PER3 & 1.000045 & 0.999185 & 1.000906 & 0.917624 \\
\hline PRKAA1 & 0.999719 & 0.999144 & 1.000295 & 0.338853 \\
\hline PRKAA2 & 0.997956 & 0.996135 & 0.999781 & $\underline{0.028131}$ \\
\hline RORA & 0.998475 & 0.995557 & 1.001403 & 0.306999 \\
\hline RORB & 1.001115 & 0.999436 & 1.002796 & 0.193172 \\
\hline SKP1 & 1.000189 & 0.999839 & 1.000538 & 0.289565 \\
\hline
\end{tabular}

2 Abbreviation: HR, hazard ratio; P, P value for whole; 95\% CI, 95\% confidence interval. Statistically

3 significant data were marked with bold and underline. 
Table 2 (on next page)

Table2 Cox regression analysis for progression-free survival 
1 Table2 Cox regression analysis for progression-free survival

\begin{tabular}{lllll}
\hline Variables & HR & Lower limit of 95\%CI & Upper limit of 95\% CI & p \\
\hline ARNTL & 0.99733 & 0.993508 & 1.001166 & 0.172199 \\
BTRC & 0.999311 & 0.998442 & 1.00018 & 0.120076 \\
CLOCK & 0.998806 & 0.997653 & 0.99996 & $\mathbf{0 . 0 4 2 6 1 4}$ \\
CRY1 & 1.001142 & 0.999805 & 1.00248 & 0.094108 \\
CRY2 & 0.999888 & 0.999376 & 1.0004 & 0.667062 \\
CSNK1D & 1.000072 & 0.999845 & 1.000298 & 0.534479 \\
CSNK1E & 1.000302 & 1.00003 & 1.000575 & $\mathbf{0 . 0 2 9 7 2 1}$ \\
CUL1 & 1.000777 & 0.99995 & 1.001604 & 0.065408 \\
DBP & 1.001326 & 1.000452 & 1.002201 & $\mathbf{0 . 0 0 2 9 3 1}$ \\
FBXL21 & 0.968937 & 0.929841 & 1.009677 & 0.133184 \\
FBXL3 & 0.999498 & 0.998974 & 1.000022 & 0.060412 \\
NFIL3 & 1.000149 & 0.999917 & 1.000381 & 0.20746 \\
NR1D1 & 1.000643 & 0.999837 & 1.001451 & 0.118107 \\
NR1D2 & 1.000433 & 1.000016 & 1.00085 & $\underline{\mathbf{0 . 0 4 1 8 8 4}}$ \\
PER1 & 0.999981 & 0.999866 & 1.000096 & 0.747887 \\
PER2 & 1.000206 & 0.999777 & 1.000635 & 0.346838 \\
PER3 & 1.000287 & 0.999887 & 1.000687 & 0.159504 \\
PRKAA1 & 0.999804 & 0.999484 & 1.000124 & 0.230166 \\
PRKAA2 & 0.998926 & 0.998031 & 0.999822 & $\underline{\mathbf{0 . 0 1 8 8 3 7}}$ \\
RORA & 1.000184 & 0.998629 & 1.001742 & 0.816508 \\
RORB & 1.00105 & 1.000425 & 1.001674 & $\mathbf{0 . 0 0 0 9 8}$ \\
SKP1 & 1.000025 & 0.999834 & 1.000217 & 0.79528 \\
\hline Abbrit & & $95 \%$ &
\end{tabular}

2 Abbreviation: HR, hazard ratio; P, P value for whole; 95\% CI, 95\% confidence interval. 
Table 3 (on next page)

Table3 Cox regression analysis for overall survival 
1 Table3 Cox regression analysis for overall survival

\begin{tabular}{|c|c|c|c|c|}
\hline Variables & HR & Lower limit of $95 \%$ CI & Upper limit of $95 \%$ CI & $\mathbf{p}$ \\
\hline ARNTL & 1.002023 & 0.9902 & 1.013987 & 0.738608 \\
\hline BTRC & 0.999165 & 0.996398 & 1.00194 & 0.554846 \\
\hline CLOCK & 0.997808 & 0.993549 & 1.002084 & 0.314471 \\
\hline CRY1 & 0.999584 & 0.995035 & 1.004154 & 0.858089 \\
\hline CRY2 & 1.000068 & 0.998619 & 1.001518 & 0.926872 \\
\hline CSNK1D & 1.001029 & 1.000437 & 1.001622 & $\underline{0.000651}$ \\
\hline CSNK1E & 0.999911 & 0.998903 & 1.000919 & 0.862001 \\
\hline CUL1 & 1.000169 & 0.997174 & 1.003174 & 0.912052 \\
\hline DBP & 0.997995 & 0.993311 & 1.002702 & 0.403177 \\
\hline FBXL21 & 0.853599 & 0.623965 & 1.167744 & 0.322149 \\
\hline FBXL3 & 1.000268 & 0.99868 & 1.001858 & 0.741299 \\
\hline NFIL3 & 0.999673 & 0.998706 & 1.000642 & 0.508415 \\
\hline NR1D1 & 0.997856 & 0.993634 & 1.002095 & 0.321049 \\
\hline NR1D2 & 0.999837 & 0.998527 & 1.001149 & 0.807904 \\
\hline PER1 & 0.999599 & 0.998984 & 1.000215 & 0.201657 \\
\hline PER2 & 0.999363 & 0.997433 & 1.001297 & 0.518096 \\
\hline PER3 & 0.999276 & 0.997557 & 1.000999 & 0.410001 \\
\hline PRKAA1 & 0.999791 & 0.998838 & 1.000744 & 0.666887 \\
\hline PRKAA2 & 1.000341 & 0.998365 & 1.002321 & 0.735204 \\
\hline RORA & 0.997031 & 0.991089 & 1.003009 & 0.329597 \\
\hline RORB & 0.995276 & 0.987314 & 1.003302 & 0.247869 \\
\hline SKP1 & 0.99986 & 0.999268 & 1.000452 & 0.642982 \\
\hline
\end{tabular}

2 Abbreviation: HR, hazard ratio; P, P value for whole; 95\% CI, 95\% confidence interval. Statistically

3 significant data were marked with bold and underline. 